\title{
ASYMPTOTIC NORMALITY OF $b$-ADDITIVE FUNCTIONS ON POLYNOMIAL SEQUENCES IN CANONICAL NUMBER SYSTEMS
}

\author{
MANFRED G. MADRITSCH
}

\begin{abstract}
We consider $b$-additive functions $f$ where $b$ is the base of a canonical number system in an algebraic number field. In particular, we show that the asymptotic distribution of $f(p(z))$ with $p$ a polynomial running through the integers of the algebraic number field is the normal law. This is a generalization of results of Bassily and Katai (for the integer case) and of Gittenberger and Thuswaldner (for the Gaussian integers).
\end{abstract}

\section{INTRODUCTION}

The objective of this paper is the consideration of additive functions in number systems. We start with a simple example of a $q$-additive function: Let $s_{q}$ denote the sum-of-digits function in base $q$, where $q$ is a positive integer. This function has been studied by several authors and we want to mention Delange [4]. He computed the average of the sum-of-digits function, i.e.,

$$
\frac{1}{N} \sum_{n \leq N} s_{q}(n)=\frac{q-1}{2} \log _{q}(N)+\gamma_{1}\left(\log _{q}(N)\right),
$$

where $\log _{q}$ denotes the logarithm in base $q$ and $\gamma_{1}$ is a continuous function of period 1 .

A canonical question is the distribution into residue classes of the sum-of-digits functions. Thus we consider sets of the form

$$
S_{r, m}(N)=\left\{n \leq N: s_{q}(n) \equiv r \bmod m\right\} .
$$

Then Mauduit and Sárközy [23] where able to show the following.

Theorem. Let $\mathcal{A}, \mathcal{B} \subset\{1, \ldots, N\}$ with $N \in \mathbb{N}$. Then the estimate

$$
\left|\#\left\{(a, b) \in \mathcal{A} \times \mathcal{B}: a+b \in S_{r, m}(2 N)\right\}-\frac{|\mathcal{A}||\mathcal{B}|}{m}\right|=\mathcal{O}\left(N^{\theta}(|\mathcal{A}||\mathcal{B}|)^{\frac{1}{2}}\right)
$$

holds, where $\theta<1$ and the implied $\mathcal{O}$-constant is absolute.

An extension of the results above to general $q$-additive functions was given by Bassily and Katai [2]. Recall that a function $f$ is said to be $q$-additive if it acts only on the $q$-adic digits, i.e., $f(0)=0$ and

$$
f(n)=\sum_{k \geq 0} f\left(a_{k}(n) q^{k}\right) \quad \text { for } \quad n=\sum_{k \geq 0} a_{k}(n) q^{k},
$$

where $a_{k}(n) \in \mathcal{N}:=\{0, \ldots, q-1\}$ are the digits of the $q$-adic expansion. Obviously, $s_{q}$ is a special $q$-additive function.

The above mentioned distributional result by Bassily and Katai [2] reads as follows.

Theorem. Let $f$ be a q-additive function such that $f\left(a q^{k}\right)=\mathcal{O}(1)$ as $k \rightarrow \infty$ and $a \in \mathcal{N}$. Furthermore let

$$
m_{k, q}:=\frac{1}{q} \sum_{a \in \mathcal{N}} f\left(a q^{k}\right), \quad \sigma_{k, q}^{2}:=\frac{1}{q} \sum_{a \in \mathcal{N}} f^{2}\left(a q^{k}\right)-m_{k, q}^{2},
$$

Date: October 27, 2008.

2000 Mathematics Subject Classification. 11K16 (11A63,60F05).

Key words and phrases. additive functions, canonical number systems, exponential sums.

Supported by the Austrian Research Foundation (FWF), Project S9603, that is part of the Austrian Research Network "Analytic Combinatorics and Probabilistic Number Theory". 
and

$$
M_{q}(x):=\sum_{k=0}^{N} m_{k, q}, \quad D_{q}^{2}(x)=\sum_{k=0}^{N} \sigma_{k, q}^{2}
$$

with $N=\left[\log _{q} x\right]$. Assume that $D_{q}(x) /(\log x)^{1 / 3} \rightarrow \infty$ as $x \rightarrow \infty$ and let $p(x)$ be a polynomial with integer coefficients, degree $d$ and positive leading term. Then, as $x \rightarrow \infty$,

$$
\frac{1}{x} \#\left\{n<x \mid \frac{f(p(n))-M_{q}\left(x^{d}\right)}{D_{q}\left(x^{d}\right)}<y\right\} \rightarrow \Phi(y),
$$

where $\Phi$ is the normal distribution function.

Results of similar type for different number systems related to substitution automaton where considered by Dumont and Thomas [8]. For distribution results of additive functions defined over number systems based on linear recurrences we refer the reader to Drmota and Gajdosik [5].

In this paper we focus on generalizations of number systems to algebraic number fields. These number systems are called canonical number systems (CNS). We shortly recall their definition following $[26,30]$.

Definition 1.1. Let

$$
m(x):=b_{n} x^{n}+b_{n-1} x^{n-1}+\cdots+b_{0} \in \mathbb{Z}[x]
$$

be such that $n \geq 1$ and $b_{n}=1$. Furthermore let $\mathcal{N}=\left\{0,1, \ldots,\left|b_{0}\right|-1\right\}$ and $\mathcal{R}$ be the quotient ring

$$
\mathcal{R}=\mathbb{Z}[x] / p(x) \mathbb{Z}[x] .
$$

- We say that a $g \in \mathcal{R}$ has a finite representation if it admits a representation of the form

$$
g=a_{0}+a_{1} x+\cdots+a_{h} x^{h}
$$

with $a_{i} \in \mathcal{N}$ for $0 \leq i \leq h$ and $d_{h} \neq 0$ for $h \neq 0$.

- The numbers $a_{i}=a_{i}(g)$ are called the digits of $g$ with respect to $(m(x), \mathcal{N})$.

- The pair $(m(x), \mathcal{N})$ is called a canonical number system $(\mathrm{CNS})$ in $\mathcal{R}$, if each $g \in \mathcal{R}$ has a finite representation. Furthermore we call $\mathcal{N}$ its digit set.

- If $m(x)$ is irreducible, then let $b$ be one of its zeros. In this case $\mathcal{R}$ is isomorphic to $\mathbb{Z}[b]$. Therefore we may replace $x$ by $b$ in the above expansions. In this case, we simplify the notation $(m(x), \mathcal{N})$ to $(b, \mathcal{N})$ and $b$ is called the base of this CNS.

Throughout this paper we are mainly interested in number systems in algebraic number fields. Let $K$ be an algebraic number field and denote by $\mathcal{O}_{K}$ its ring of integers. Furthermore let $\mathrm{N}$ and Tr denote the norm and trace of an element of $K$ over $\mathbb{Q}$, respectively. Then for $b \in \mathcal{O}_{K}$ we set $\mathcal{N}:=\{0,1, \ldots, N(b)-1\}$ and get that $(b, \mathcal{N})$ is a number system if the minimal polynomial $m_{b}(x)$ of $b$ over $\mathbb{Q}$ satisfies the conditions of $m(x)$ above.

When considering number systems one is interested in certain properties, first of all, which are the possible bases. This has been answered in a series of papers $[1,3,14,15,16]$. Secondly one has to face effects such as amenability, i.e., there exist two different expansions of one number. In fact, one can construct a graph which characterizes all the amenable expansions. This has been done by Müller et al. [25] (with a direct approach) and by Scheicher and Thuswaldner [29] (consideration of the odometer).

Another view on number systems are normal numbers. These are numbers in which expansion every possible block occurs asymptotically equally often. Constructions of such numbers have been considered by Dumont et al. [7] and the author in [21, 22]

In this paper we mainly concentrate on additive functions. These are functions that act only on the digits of an expansion. Thus we define additive functions in these number systems as follows.

Definition 1.2. Let $(b, \mathcal{N})$ be a CNS over $\mathcal{O}_{K}$. A function $f$ is called b-additive if $f(0)=0$ and for $g \in \mathcal{O}_{K}$

$$
f(g)=\sum_{k \geq 0} f\left(a_{k}(g) b^{k}\right), \quad \text { for } g=\sum_{k \geq 0} a_{k}(g) b^{k} \quad\left(a_{k}(g) \in \mathcal{N}\right) .
$$


As indicated above the simplest version of an additive function is the sum-of-digits function $s_{b}$ defined by

$$
s_{b}(g):=\sum_{k \geq 0} a_{k}(g)
$$

A first step towards CNS is the consideration of $b$-additive function in the field of Gaussian rationals. First a generalization of the result by Delange was given by Grabner et al. in [12].

Theorem. Let $b=-n \pm i$ be a base of a CNS in $\mathbb{Z}[i]$. Then we have

$$
\sum_{\substack{\mathrm{N}(z) \leq N \\ z \in \mathbb{Z}[i]}} s_{b}(z)=\frac{\mathrm{N}(b)-1}{2} \pi N \log _{\mathrm{N}(b)} N+N \gamma_{2}\left(\log _{\mathrm{N}(b)} N\right)+\mathcal{O}\left(\sqrt{N} \log _{\mathrm{N}(b)} N\right)
$$

where $\gamma_{2}$ is a continuous function of period 1.

Also the result by Bassily and Katai was generalized to number systems over the Gaussian rationals. Gittenberger and Thuswaldner [11] gained the following distribution result.

Theorem. Let $(b, \mathcal{N})$ be a canonical number system over $\mathbb{Z}[i]$. Let $f$ be a b-additive function such that $f\left(c b^{k}\right)=\mathcal{O}(1)$ as $k \rightarrow \infty$ and $c \in \mathcal{N}$. Furthermore let

$$
m_{k, b}:=\frac{1}{\mathrm{~N}(b)} \sum_{c \in \mathcal{N}} f\left(c b^{k}\right), \quad \sigma_{k, b}^{2}:=\frac{1}{\mathrm{~N}(b)} \sum_{c \in \mathcal{N}} f^{2}\left(c b^{k}\right)-m_{k, b}^{2},
$$

and

$$
M_{b}(x):=\sum_{k=0}^{L} m_{k, q}, \quad D_{b}^{2}(x)=\sum_{k=0}^{L} \sigma_{k, q}^{2}
$$

with $L=\left[\log _{\mathrm{N}(b)} x\right]$. Assume that $D_{b}(x) /(\log x)^{1 / 3} \rightarrow \infty$ as $x \rightarrow \infty$ and let $p(x)$ be a polynomial of degree $d$ with coefficients in $\mathbb{Z}[i]$. Then, as $N \rightarrow \infty$,

$$
\frac{1}{\#\{z \in \mathbb{Z}[i] \mid \mathrm{N}(z)<N\}} \#\left\{\mathrm{~N}(z)<N \mid \frac{f(p(z))-M_{b}\left(N^{d}\right)}{D_{b}\left(N^{d}\right)}<y\right\} \rightarrow \Phi(y),
$$

where $\Phi$ is the normal distribution function and $z$ runs over the Gaussian integers.

This build the base for further considerations of $b$-additive functions over CNS in general. First the result of Delange was further generalized to arbitrary number fields by Thuswaldner [32]. Furthermore the moments of the sum-of-digits function in algebraic number fields were considered by Gittenberger and Thuswaldner [10].

Theorem. Let $K$ be a number field of degree $n$ and $\mathcal{O}_{K}$ its ring of integers. Furthermore, let $b$ be a base of a CNS. Then

$$
\begin{aligned}
\sum_{z \in P(N)}\left(s_{b}(z)\right)^{d}= & c_{b}\left(\frac{|\mathrm{N}(b)|-1}{2}\right)^{d} N \log _{|\mathrm{N}(b)|}^{d} N+N \sum_{j=0}^{d-1} \log _{|\mathrm{N}(b)|}^{j} N \gamma_{j}\left(\log _{|\mathrm{N}(b)|} N\right) \\
& +\mathcal{O}\left(N^{\frac{n-1}{n}} \log _{|\mathrm{N}(b)|}^{d} N\right),
\end{aligned}
$$

where $c_{b}$ is a constant depending on $K$ and $b$ and the $\gamma_{j}$ s are continuous functions of period 1.

In the same vain the above mentioned result by Mauduit and Sárközy was generalized to arbitrary number fields by Thuswaldner [33]. Therefore we write

$$
U_{r, m}(M(T))=\left\{z \in M(T): s_{b}(z) \equiv r \bmod m\right\},
$$

where $M(T)$ is the set described below in (2.2). Then his result reads as follows.

Theorem. Let $K$ be a number field of degree $n$ with ring of integers $\mathcal{O}_{K}$. Let $b$ be the base of a canonical number system in $\mathcal{O}_{K}$ and $m_{b}(x)=x^{n}+\cdots+b_{1} x+b_{0}$ the minimal polynomial of $b$. If $\left(m_{b}(1), m\right)=1$, then

$$
\left|\#\left\{(a, b) \in \mathcal{A} \times \mathcal{B}: a+b \in S_{r, m}(M(2 T))\right\}-\frac{|\mathcal{A}||\mathcal{B}|}{m}\right|=\mathcal{O}\left(|M(T)|^{\theta}(|\mathcal{A}||\mathcal{B}|)^{\frac{1}{2}}\right)
$$


holds for any two sets $\mathcal{A}, \mathcal{B} \subset M(T)$. Furthermore $\theta<1$ and the implied $\mathcal{O}$-constant is absolute.

Despite of these considerations of the sum-of-digits function and other $b$-additive functions, we also want to mention Katai and Liardet [17], who could show a Delange type result for $b$-multiplicative functions. Finally there has also been work on the generalization of Waring's Problem restricted to sets of the form $S_{r, m}$ defined above. Here we want to mention Pethö and Tichy [27] (counting the number of solutions for a $S$-unit equation) and Thuswaldner and Tichy [34] (counting the number of solutions of Waring's Problem with digital restrictions).

In the present paper our aim is to extend the result of Bassily and Katai and Gittenberger and Thuswaldner to arbitrary algebraic number fields. The main problem we have to face is the different setting for number systems. Especially the fact that the conjugates of a number need not to have equal absolute value will play a role. This difference occurs essentially in the estimation of the involved Weyl sums. Know estimates deal with Waring's problem and its generalization. Since in these generalizations the leading coefficients of the involved polynomial go through the whole unit interval and its approximations therefore only targets the splitting into major and minor arcs, we need a different approach. In Section 3 we will develop a more general estimation of these sums.

\section{Definitions and Results}

In the following paragraphs we will define the tools we need in order to properly estimate the distribution. These definitions are standard in the area and the reader may refer to Ribenboim [28] or Wang [35].

Throughout the rest of the paper we fix an algebraic number field $K$ and denote by $\mathcal{O}_{K}$ its ring of integers. Then let $K^{(\ell)}\left(1 \leq \ell \leq r_{1}\right)$ be the real conjugates of $K$, while $K^{(m)}$ and $K^{\left(m+r_{2}\right)}$ $\left(r_{1}<m \leq r_{1}+r_{2}\right)$ are the complex conjugates of $K$, where $r_{1}+2 r_{2}=n$. Throughout this paper the indices $\ell$ and $m$ are always over the sets of integers cited here. Furthermore we set $r=r_{1}+r_{2}$ and call the pair $\left(r_{1}, r_{2}\right)$ the signature of $K$.

If not stated otherwise an upper case letter will always denote a real number, a lower case letter an element of $\mathcal{O}_{K}$ and a Greek letter an element of $\bar{K}$, the completition of $K$. Furthermore sums are always extended over rational or algebraic integers, respectively.

For $\gamma \in K$ we denote by $\gamma^{(i)}(1 \leq i \leq n)$ the conjugates of $\gamma$. In order to extend the term of conjugation to the completition $\bar{K}$ of $K$ we define for $\gamma_{j} \in K$ and $x_{j} \in \mathbb{R}(1 \leq j \leq n)$ $\lambda=\sum_{1 \leq j \leq n} x_{j} \gamma_{j}$ and $\lambda^{(i)}:=\sum_{1 \leq j \leq n} x_{j} \gamma_{j}^{(i)}$. We recall that for $\lambda \in \bar{K}$

$$
\mathrm{N}(\lambda)=\prod_{1 \leq i \leq n} \lambda^{(i)}, \quad \operatorname{Tr}(\lambda)=\sum_{1 \leq i \leq n} \lambda^{(i)}, \quad|\lambda|=\max _{1 \leq i \leq n}\left|\lambda^{(i)}\right|
$$

are the norm, trace and house of an element of $\bar{K}$ over $\mathbb{Q}$, respectively. Furthermore for $\lambda \in \bar{K}$ let

$$
e(x):=\exp (2 \pi i x) \text { and } E(\lambda)=e(\operatorname{Tr}(\lambda)) .
$$

Let $\delta$ be the different and $D$ the absolute value of the discriminant of $K$. We need some geometry of numbers and therefore let $\omega_{1}, \ldots, \omega_{n}$ be an integral basis of $\mathcal{O}_{K}$ and let $\rho_{1}, \ldots, \rho_{n}$ be the corresponding basis of $\delta^{-1}$ such that

$$
\operatorname{Tr}\left(\rho_{i} \omega_{j}\right)= \begin{cases}1 & i=j \\ 0 & i \neq j .\end{cases}
$$

Let $\Lambda$ be the lattice constructed by the $n$ linear forms

$$
L_{i}=\sum_{j=1}^{n} \rho_{j}^{(i)} x_{j}
$$

Then we denote by $\lambda_{1}$ the first successive minimum of the convex body $B:=\left\{z \in \mathbb{R}^{n}:|z| \leq 1\right\}$ with respect to the lattice $\Lambda$. 
We call a number totally non-negative if $\lambda^{(i)} \geq 0$ for $1 \leq i \leq n$. As we will successively extend the maximum length of the expansions we define $M\left(T_{1}, \ldots, T_{r}\right)$ to be the set

$$
M\left(T_{1}, \ldots, T_{r}\right):=\left\{\eta \in \mathcal{O}_{K}:\left|\eta^{(i)}\right| \leq T_{i}, 1 \leq i \leq r\right\} .
$$

Furthermore we will write for short $M(T):=M(T, \ldots, T)$.

Now we can state our main result.

Theorem 2.1. Let $(b, \mathcal{N})$ be a $C N S$ and $f$ be a b-additive function such that $f\left(a b^{k}\right)=\mathcal{O}(1)$ as $k \rightarrow \infty$ and $a \in \mathcal{N}$. Furthermore let

$$
m_{k, b}:=\frac{1}{\mathrm{~N}(b)} \sum_{a \in \mathcal{N}} f\left(a b^{k}\right), \quad \sigma_{k, b}^{2}:=\frac{1}{\mathrm{~N}(b)} \sum_{a \in \mathcal{N}} f^{2}\left(a b^{k}\right)-m_{k, b}^{2},
$$

and

$$
M_{b}(x):=\sum_{k=0}^{L} m_{k, q}, \quad D_{b}^{2}(x)=\sum_{k=0}^{L} \sigma_{k, q}^{2}
$$

with $L=\left[\log _{\mathrm{N}(b)} x\right]$. Assume that there exists an $\varepsilon>0$ such that $D_{b}(x) /(\log x)^{\varepsilon} \rightarrow \infty$ as $x \rightarrow \infty$ and let $p \in \mathcal{O}_{K}[X]$ be a polynomial of degree $d$. Then, as $T \rightarrow \infty$,

$$
\frac{1}{\# M(T)} \#\left\{z \in M(T) \mid \frac{f(p(z))-M_{b}\left(T^{d}\right)}{D_{b}\left(T^{d}\right)}<y\right\} \rightarrow \Phi(y),
$$

where $\Phi$ is the normal distribution function.

We will show this theorem in five steps.

(1) In Section 3 we start with the estimation of the Weyl sums which will occur in the proof. Therefore we use tools which come from generalizations of Waring's Problem to algebraic number fields together with Hua's method of estimating Weyl sums. The main difference will be the approximation of the coefficients we use.

(2) For counting the number of occurrences of a certain digit in the expansion of the integer values of the polynomial $p$ we need an Urysohn function. This function is developed in Section 4 were we consider the fundamental domain of the CNS.

(3) The error term when counting the number of occurrences of a digit comes from those near the border of the fundamental domain. Therefore we estimate the number of elements which are in a small tube around the border in Section 5.

(4) In Section 6 we have to count the number of elements within the fundamental domain itself, which gives the proof of the central proposition.

(5) Finally we show Theorem 2.1 by truncation and two applications of the Fréchet-Shohat Theorem.

\section{WEYL SUMS}

In this section we want to consider and estimate the exponential sums which will occur in the following sections. We will begin by giving some background on how exponential sums are defined in number fields.

Throughout this section let

$$
g(x)=\alpha_{d} x^{d}+\cdots+\alpha_{1} x
$$

be a polynomial of degree $d$. Let a boldface letter always denote a vector. Then for $\mathbf{T}=\left(T_{1}, \ldots, T_{r}\right)$ we set $M(\mathbf{T})=M\left(T_{1}, \ldots, T_{r}\right)$. The sum

$$
S(g, \mathbf{T})=\sum_{x \in M(\mathbf{T})} E(g(x))
$$

where $E$ is as in (2.1), is called a Weyl sum.

The main tool in order to estimate these sums is Weyl's differentiation method. Therefore we need a generalization of Dirichlet's theorem on rational approximation, which is provided by Siegel (cf. [31]). Since in our case the $T_{i}$ are not all equal (see Lemma 6.1) we have to slightly modify Siegel's original theorem in order to cope with this new situation. 
Lemma 3.1. Let $N_{1}, \ldots, N_{r}$ be real numbers and let $N=\sqrt[n]{N_{1} \cdots N_{r_{1}}\left(N_{r_{1}+1} \cdots N_{r_{1}+r_{2}}\right)^{2}}$ be their geometric mean. Suppose that $N>D^{\frac{1}{n}}$, then, corresponding to any $\xi \in K$, there exist $q \in \mathcal{O}_{K}$ and $a \in \delta^{-1}$ such that

$$
\begin{gathered}
\left|q^{(i)} \xi^{(i)}-a^{(i)}\right|<N_{i}^{-1}, \quad 0<\left|q^{(i)}\right| \leq N_{i}, \quad 1 \leq i \leq r, \\
\max \left(N_{i}\left|q^{(i)} \xi^{(i)}-a^{(i)}\right|,\left|q^{(i)}\right|\right) \geq D^{-\frac{1}{2}}, \quad 1 \leq i \leq r,
\end{gathered}
$$

and

$$
\mathrm{N}((q, a \delta)) \leq D^{\frac{1}{2}} .
$$

Proof. This easily follows from the appropriate modifications in the proof of Theorem 3.1 of [35].

Now we can state the main estimate of the exponential sum above.

Proposition 3.2. Let

$$
T=\sqrt[n]{T_{1} \cdots T_{r_{1}}\left(T_{r_{1}+1} \cdots T_{r_{1}+r_{2}}\right)^{2}}
$$

be the geometric mean of the $T_{i}(1 \leq i \leq r)$. Suppose that $\alpha_{k}$ satisfies the hypotheses from Lemma 3.1 with $N_{i}=T_{i}^{d}(\log T)^{-\sigma_{1}}$ and

$$
(\log T)^{\sigma_{1}} \leq \alpha_{k}^{(i)} \leq T_{i}^{d}(\log T)^{-\sigma_{1}} \quad 1 \leq i \leq r,
$$

then

with $\sigma_{1} \geq 2^{d-1}\left(\sigma_{0}+r 2^{2 d}\right)$.

$$
S(g, \mathbf{T}) \ll T^{n}(\log T)^{-\sigma_{0}}
$$

The proof consists of modifications of the proof of Theorem 3.2 of Wang [35], since in our considerations the $T_{i}$ need not all be equal and we essentially need the $(\log T)^{-\sigma_{0}}$ term. We start with the main tools needed for the proof of Proposition 3.2.

Lemma 3.3 ([35, Lemma 3.2]). Let $T_{i}(1 \leq i \leq r)$ be positive integers and set $T_{r_{1}+r_{2}+i}=T_{r_{1}+i}$ for $\left(1 \leq i \leq r_{2}\right)$. Then

$$
M(T)=\frac{2^{r} \pi^{r_{2}}}{\sqrt{D}} T_{1} \cdots T_{n}+\mathcal{O}\left(T_{0}^{n-1}\right),
$$

where $T_{0}=\max \left(1,\left(T_{1} \cdots T_{n}\right)^{1 / n}\right)$.

Since in the classical case the $T_{i}$ are all equal we have to rewrite the proof of Wang's Lemma 3.6. Therefore we need the following adoption of Lemma 3.5 of Wang [35].

Lemma 3.4. Let $T_{i}$ and $N_{i} \geq 0$ for $1 \leq i \leq s$ be integers. Then denote by $M$ the set of all points $\left(t_{1}, \ldots, t_{s}\right) \in \mathbb{Z}^{s}$ such that

$$
T_{i} \leq t_{i}<T_{i}+N_{i} \quad 1 \leq i \leq s .
$$

Let $M_{0}$ be a subset of $M$ and define

$$
S=\sum_{\mathbf{t} \in M_{0}} \min \left(\frac{N_{1}}{t_{1}}, \cdots, \frac{N_{s}}{t_{s}}\right) .
$$

Then

$$
S \ll N\left(\# M_{0}\right)^{1-\frac{1}{s}}(\log (N+2)),
$$

where $N$ is the geometric mean of the $N_{i}$.

Proof. This proof mainly follows that of Lemma 3.5 of Wang [35]. In the same way we start by setting $M_{\nu}$ to be the subset of $M_{0}$ such that $\frac{t_{\nu}}{N_{\nu}} \geq \frac{t_{i}}{N_{i}}$ for $1 \leq i \leq s$. Furthermore we denote by $A_{\nu}=\# M_{\nu}$ its number of elements and by

$$
S_{\nu}=\sum_{\mathbf{t} \in M_{\nu}} \min \left(\frac{N_{1}}{t_{1}}, \cdots, \frac{N_{s}}{t_{s}}\right)
$$


the restriction of the sum $S$ to elements of $M_{\nu}$.

Then it suffices to show that

$$
S_{\nu} \ll N A_{\nu}^{1-\frac{1}{s}} \log \left(N_{\nu}+2\right),
$$

which together with $S \leq S_{1}+\cdots+S_{s}$ proves the lemma.

Without loss of generality we show this for $\nu=1$. For $t>0$ let $D(t)$ be the subset of $\mathbf{u} \in \mathbb{R}^{s}$ such that

$$
t \geq u_{1}>0, \quad \frac{u_{1}}{N_{1}} \geq \frac{u_{i}}{N_{i}} \geq 0, \quad 2 \leq i \leq s .
$$

Let $M(t):=D(t) \cap \mathbb{Z}^{s}$ be the integer points in $D(t)$ and denote by $n(t)$ their number. In the same manner let $M_{0}(t):=\left\{\mathbf{t} \in M_{0}: t \geq t_{1}\right\}$ and denote by $n_{0}(t)=\# M_{0}(t)$ its cardinality.

Now let $t_{0}$ be an integer such that

$$
n\left(t_{0}\right) \leq A_{1}=n_{0}\left(N_{1}\right)<n\left(t_{0}+1\right) .
$$

Then

$$
S_{1} \leq \sum_{\mathbf{t} \in M\left(t_{0}+1\right)} \frac{N_{1}}{t_{1}} \leq N_{1} \prod_{i=2}^{s} \frac{N_{i}}{N_{1}}\left(t_{0}+2\right) \sum_{t_{1} \leq t_{0}+1} \frac{1}{t_{1}} \leq \frac{N^{s}\left(t_{0}+2\right)^{s-1}}{N_{1}^{s-1}} \log \left(t_{0}+2\right)
$$

and

$$
A_{1} \geq n\left(t_{0}\right) \geq \sum_{t=1}^{t_{0}} \prod_{i=2}^{s} \frac{N_{i}}{N_{1}} t=\frac{N^{s}}{N_{1}^{s}} \sum_{t=1}^{t_{0}} t^{s-1} \geq c(s) \frac{N^{s}}{N_{1}^{s}} t_{0}^{s} .
$$

Putting these together proves the lemma.

Now we state our modified version of Lemma 3.6 of Wang [35]. In its original version this lemma essentially goes back to Mitsui [24].

Lemma 3.5. Let $A, B_{i}(1 \leq i \leq r), N$ be positive numbers satisfying $A \geq 1$ and $N \gg 1$. Furthermore let $B$ be the geometric mean of the $B_{i}$ and let $N_{i}(1 \leq i \leq r)$ be in the same ration to $N$ as the $B_{i}$ are to $B$, i.e.,

$$
B:=\sqrt[n]{B_{1} \cdots B_{r_{1}}\left(B_{r_{1}+1} \cdots B_{r}\right)^{2}} \quad \text { and } \quad N_{i}:=N \frac{B_{i}}{B} \quad 1 \leq i \leq r .
$$

Suppose that $1 \leq B \ll N$, then, for any $\xi$,

$$
\sum_{m \in M(\mathbf{B})} \min _{1 \leq i \leq n}\left(A,\left|1-E\left(\xi m \omega_{i}\right)\right|^{-1}\right) \ll A B^{n}\left(\frac{1}{|\mathrm{~N}(q)|}+\frac{1}{B}+\frac{N \log N}{A B}+\frac{\log N}{A}\right),
$$

where $q$ denotes an integer satisfying the conditions in Lemma 3.1 with $\xi$ and $N_{i}$.

Proof. Since this is a modification of the proof of Lemma 3.6 in Wang [35] we only sketch the proof and mainly follow the lines there.

First let $\mathbf{X}$ be the Minkowski embedding, i.e., for $\xi \in K$

$$
\mathbf{X}(\xi)=\left(X_{1}(\xi), \ldots, X_{n}(\xi)\right):=\left(\xi^{(1)}, \ldots, \xi^{\left(r_{1}\right)}, \Re\left(\xi^{\left(r_{1}+1\right)}\right), \ldots, \Im\left(\xi^{\left(r_{1}+r_{2}\right)}\right)\right) .
$$

Then we write with rational integers $e_{i}$ and $-\frac{1}{2} \leq d_{i} \leq \frac{1}{2}$

$$
\operatorname{Tr}\left(\xi m \omega_{i}\right)=e_{i}+d_{i} \quad(1 \leq i \leq n) \quad \text { and set } \quad \zeta=\sum_{i=1}^{n} d_{i} \rho_{i}
$$

One easily checks that

$$
S=\sum_{m \in M(\mathbf{B})} \min _{1 \leq i \leq n}\left(A,\left|1-E\left(\xi m \omega_{i}\right)\right|^{-1}\right) \ll \sum_{m \in M(\mathbf{B})} \min _{1 \leq i \leq n}\left(A,\left|X_{i}(\zeta)\right|^{-1}\right)=S^{*} .
$$

Now we assign to every $m$ in $M(\mathbf{B})$ its corresponding $\zeta$ and a vector $y(m)$ defined by

$$
y(m)=\left(R_{1} X_{1}(\zeta), \ldots, R_{n} X_{n}(\zeta)\right) \text { with } \quad R_{i}=2 D^{\frac{1}{n}}\left|q^{(i)}\right| .
$$


We divide the set $\{1,2, \ldots, n\}$ into three parts

$$
\begin{aligned}
& J_{1}:=\left\{1 \leq i \leq n: \frac{B_{i}}{N_{i}} D^{\frac{1}{n}} \geq 2 c_{11}\left|q^{(i)}\right|\right\} \\
& J_{2}:=\left\{1 \leq i \leq n: \frac{1}{2} \geq 2 c_{11}\left|q^{(i)}\right|>\frac{B_{i}}{N_{i}} D^{\frac{1}{n}}\right\}, \\
& J_{3}:=\left\{1 \leq i \leq n: 2 c_{11}\left|q^{(i)}\right|>\frac{1}{2}\right\} .
\end{aligned}
$$

Furthermore we set

$$
\tau_{i}=2 \frac{B_{i}}{N_{i}} D^{\frac{1}{n}} \text { for } i \in J_{1}, \quad \tau_{i}=4 c_{11}\left|q^{(i)}\right| \text { for } i \in J_{2} .
$$

For the rest we set $\tau_{i}$ such that $\prod_{i=1}^{n} \tau_{i}=2^{-2 n}$. Then we divide the set of possible vectors $y(m)$ by defining for every vector $\mathrm{g} \in \mathbb{Z}^{n}$

$$
B(\mathbf{g}):=\left\{\mathbf{x}: \tau_{i}\left(g_{i}-\frac{1}{2}\right) \leq x_{i}<\tau_{i}\left(g_{i}+\frac{1}{2}\right), 1 \leq i \leq n\right\} .
$$

By the same lines as in the proof of Wang we get that if there are two $m$ and $m_{1}$ such that $m, m_{1} \in B(\mathbf{g})$ then $m-m_{1} \in \mathfrak{a}$ for a certain ideal $\mathfrak{a}$ with

$$
|\mathrm{N}(q)| \leq D^{\frac{1}{n}} \mathrm{~N}(\mathfrak{a}) .
$$

Finally we denote by $W(\mathbf{g})$ the number of $m \in M(\mathbf{B})$ such that $y(m) \in B(\mathbf{g})$. Thus following the lines of the proof of Wang we get that

$$
W(\mathbf{g}) \ll 1+W_{0}=1+\prod_{i \in J_{1}} N_{i} \prod_{j \in J_{2} \cup J_{3}} \frac{B_{j}}{\left|q^{(j)}\right|}=1+B^{n} \prod_{i \in J_{1}} \frac{N_{i}}{B_{i}} \prod_{j \in J_{2} \cup J_{3}}\left|q^{(j)}\right|^{-1} .
$$

Now we split the sum $S^{*}$ up into two parts where $S_{1}$ consists of all elements $m \in M(\mathbf{B})$ and $y(m) \in B(\mathbf{0})$ and $S_{2}$ is the rest.

We start with the estimation of $S_{1}$ and distinguish two cases according to whether $J_{1} \cup J_{2}=\emptyset$ or not.

- For $J_{1} \cup J_{2}=\emptyset$ we get as in the proof of Wang that

$$
S_{1} \ll A+\frac{A B^{n}}{|\mathrm{~N}(q)|} .
$$

- For $J_{1} \cup J_{2} \neq \emptyset$ we rewrite the sum and get

$$
S_{1} \ll \sum_{\substack{m \in \mathfrak{a} \\ m \in M(2 \mathbf{B})}} \min _{j \in J_{1} \cup J_{2}}\left(A, \frac{N_{j}}{\left|X_{j}\left(m+\xi_{0}\right)\right|}\right) .
$$

We again divide the area of possible $\mathbf{X}$. For $\mathbf{t} \in \mathbb{Z}^{n}$ we define

$$
B^{*}(\mathbf{t})=\left\{\mathbf{x}: \frac{\mathrm{N}(\mathfrak{a})}{3} \frac{B_{i}}{B}\left(t_{i}-\frac{1}{2}\right) \leq x_{i}<\frac{\mathrm{N}(\mathfrak{a})}{3} \frac{B_{i}}{B}\left(t_{i}+\frac{1}{2}\right)\right\} .
$$

We get that there is at most one element $m \in M(2 \mathbf{B})$ for every $\mathbf{t} \in \mathbb{Z}^{n}$. By noting our definition of $N_{i}$ we rewrite the sum to get

$$
S_{1} \ll \sum_{\mathbf{t}} \min _{j \in J_{1} \cup J_{2}}\left(A, \frac{N}{\left|t_{j}\right| \mathrm{N}(\mathfrak{a})^{\frac{1}{n}}}\right) \ll A B^{n}\left(\frac{1}{B}+\frac{N \log N}{A B}\right),
$$

where we used Wang's estimations since the sum is the same.

Together with the estimation (3.1) we get for $S_{1}$ that

$$
S_{1} \ll A B^{n}\left(\frac{1}{|\mathrm{~N}(q)|}+\frac{1}{B}+\frac{N \log N}{A B}\right) .
$$

Now we are left with estimating $S_{2}$. Therefore we distinguish the two cases according to whether $W_{0}>1$ or not. 
- For the first case $\left(W_{0}>1\right)$ we get by following the lines of the proof of Wang that

$$
S_{2} \ll B^{n} \log N
$$

- In the case of $W_{0} \leq 1$ we reach the estimate

$$
S_{2} \ll \sum_{\mathbf{g}_{i} \in G_{0}} \min _{i \in J_{3}}\left(\frac{N_{i}}{\left|q^{(i)}\right|}\right),
$$

where $G_{0}$ is the set of all $g_{i}, i \in J_{3}$ such that

$$
W(\mathbf{g}) \neq 0, \quad\left\{g_{i}\right\} \neq \mathbf{0} .
$$

In the same manner as in Wang's proof we get that the value $\left|g_{i}\right|$ in $G_{0}$ does not exceed $N_{i}$. Thus by an application of Lemma 3.4 we get that

$$
S_{2} \ll N B^{n-1} \log N
$$

Together with the estimation (3.3) this yields

$$
S_{2} \ll A B^{n}\left(\frac{\log N}{A}+\frac{N \log N}{A B}\right) .
$$

Putting the estimates of $S_{1}$ and $S_{2}$ in (3.2) and (3.4) together proves the lemma.

Lemma 3.6 ([35, Lemma 3.8]). Let $m \in \mathcal{O}_{K}$ and $T$ be the geometric mean of the $T_{i}$, i.e.,

$$
T=\sqrt[n]{T_{1} \cdots T_{r_{1}}\left(T_{r_{1}+1} \cdots T_{r_{1}+r_{2}}\right)^{2}}
$$

Then

$$
\sum_{h \in M(\mathbf{T})} E(\xi m h) \ll T^{n-1} \min _{1 \leq i \leq n}\left(T,\left|1-E\left(\xi m \omega_{i}\right)\right|^{-1}\right),
$$

where $\omega_{i}(1 \leq i \leq n)$ is an integral basis.

Lemma 3.7 ([35, Lemma 3.9]). Suppose that $1 \leq t \leq d-1$ and $T$ is the geometric mean of the $T_{i}$, then we have

$$
|S(g, \mathbf{T})|^{2^{t}} \ll T^{\left(2^{t}-t-1\right) n} \sum_{h_{1}, \ldots, h_{t} \in M(2 T)} \sum_{h \in M(\mathbf{T})} E\left(h_{1} \cdots h_{t} \bar{g}\left(h, h_{1}, \ldots, h_{t}\right) \xi\right),
$$

where

$$
\bar{g}\left(h, h_{1}, \ldots, h_{t}\right)=d(d-1) \cdots(d-t+1) \alpha_{d} h^{d-t}+\cdots
$$

is a polynomial of degree $d-t$ in $h, h_{1}, \ldots, h_{t}$.

Now we consider the divisor function in more detail. This idea essentially goes back to Hua [13].

Lemma 3.8 ([35, Lemma 3.7]). For $a \in \mathcal{O}_{k}$ and $T \geq 0$ let $d_{k}(a, T)$ be the number of solutions of the equation

$$
u_{1} \cdots u_{k}=a, \quad \text { where } u_{1}, \ldots, u_{k} \in M(T) \text { for } i=1, \ldots, k \text {. }
$$

Then

$$
d_{k}(a, T) \ll|\mathrm{N}(a)|^{\varepsilon}(\log T)^{(r-1)(k-1)} .
$$

We write for short $d(a, T):=d_{2}(a, T)$. Now we have collected all our tools in order to estimate sums involving divisor function, which will occur in our proof of Proposition 3.2.

Lemma 3.9. Let $t$ be a non-negative integer. Furthermore let $\mathbf{T}=\left(T_{1}, \ldots, T_{r}\right)$ and suppose that $T_{i} \ll T_{0}$ for $1 \leq i \leq r$, then

$$
\sum_{m \in M(\mathbf{T})} \frac{\left(d\left(m, T_{0}\right)\right)^{t}}{\mathrm{~N}(m)} \ll\left(n\left(\log T_{0}\right)^{r}\right)^{2^{t}}
$$


Proof. For simplicity we set $T_{r_{1}+r_{2}+i}=T_{r_{1}+i}$ for $1 \leq i \leq r_{2}$ and continue by induction on $t$. For $t=0$ we get that $m \in M(\mathbf{T})$ implies that $|\mathrm{N}(m)| \leq \prod_{i=1}^{n} T_{i} \ll T_{0}^{n}$. Thus by Lemma 3.8

$$
\sum_{m \in M(\mathbf{T})} \frac{1}{|\mathrm{~N}(m)|} \ll \sum_{N \leq T_{0}^{n}} \frac{\left(\log T_{0}\right)^{r-1}}{N} \ll n\left(\log T_{0}\right)^{r} .
$$

Now we assume that the lemma holds for $t-1$. Then

$$
\begin{aligned}
\sum_{m \in M(\mathbf{T})} \frac{\left(d\left(m, T_{0}\right)\right)^{t}}{|\mathrm{~N}(m)|} & =\sum_{m \in M(\mathbf{T})} \frac{\left(d\left(m, T_{0}\right)\right)^{t-1}}{|\mathrm{~N}(m)|} \sum_{\substack{u v=m \\
u, v \in M\left(T_{0}\right)}} 1 \leq \sum_{u \in M\left(T_{0}\right)} \sum_{\substack{u v=m \\
m \in M(\mathbf{T})}} \frac{\left(d\left(m, T_{0}\right)\right)^{t-1}}{|\mathrm{~N}(m)|} \\
& =\sum_{u \in M(\mathbf{T})} \frac{\left(d\left(u v, T_{0}\right)\right)^{t-1}}{|\mathrm{~N}(u \cdot v)|} \\
& \ll\left(n \cdot\left(\log T_{0}\right)^{r}\right)^{2^{t-1}}\left(n \cdot\left(\log T_{0}\right)^{r}\right)^{2^{t-1}}=\left(n \cdot\left(\log T_{0}\right)^{r}\right)^{2^{t}} .
\end{aligned}
$$

By the last lemma we can estimate a divisor sum which occurs in the estimation of our Weyl sum.

Lemma 3.10. Let $t$ be a non-negative integer and let $\mathbf{T}=\left(T_{1}, \ldots, T_{r}\right)$. Furthermore set $T_{r_{1}+r_{2}+i}=$ $T_{r_{1}+i}$ for $1 \leq i \leq r_{2}$ and suppose that $T_{i} \ll T_{0}$ for $1 \leq i \leq n$. Then

$$
\sum_{m \in M(\mathbf{T})}\left(d\left(m, T_{0}\right)\right)^{t} \ll T_{1} \cdots T_{n}\left(n\left(\log T_{0}\right)^{r}\right)^{2^{t}-1} .
$$

Proof. We show this by induction on $t$. For $t=0$ this essentially is Lemma 3.3. Now we assume that the lemma holds for $t-1$, then by an application of Lemma 3.9

$$
\begin{aligned}
\sum_{m \in M(\mathbf{T})}\left(d\left(m, T_{0}\right)\right)^{t} & =\sum_{m \in M(\mathbf{T})}\left(d\left(m, T_{0}\right)\right)^{t-1} \sum_{\substack{u v=m \\
u, v \in M\left(T_{0}\right)}} 1=\sum_{u \in M(\mathbf{T})} \sum_{\substack{u v=m \\
u \in M(\mathbf{T})}}\left(d\left(m, T_{0}\right)\right)^{t-1} \\
& \left.=\sum_{u \in M(\mathbf{T})}\left(u v, T_{0}\right)\right)^{t-1} \\
& \ll \sum_{u \in M\left(T_{1}\left|u^{(1)}\right|^{-1}, \ldots, T_{r}\left|u^{(r)}\right|^{-1}\right)}\left(d\left(u, T_{0}\right)\right)^{t-1} \frac{T_{1} \cdots T_{n}}{|\mathrm{~N}(u)|}\left(n\left(\log T_{0}\right)^{r}\right)^{2^{t-1}-1} \\
& \ll T_{1} \cdots T_{n}\left(n\left(\log T_{0}\right)^{r}\right)^{2^{t}-1}
\end{aligned}
$$

Finally we need a Lemma whose idea essentially goes back to Hua (c.f. Hilfssatz 6.1 of [13]) in order to get a better estimation of the Weyl sum.

Lemma 3.11. Let $t$ be a non-negative integer and let $\mathbf{T}=\left(T_{1}, \ldots, T_{r}\right)$. Suppose that $T_{i} \ll T_{0}$ for $1 \leq i \leq n$. Then, for any $\sigma_{2} \geq 2^{3 t-1}$ we get

$$
\sum_{m \in M(\mathbf{T})}^{\prime}\left(d\left(m, T_{0}\right)\right)^{t} \ll T_{0}^{n}\left(n\left(\log T_{0}\right)^{r}\right)^{-\sigma_{2}}
$$

where $\sum^{\prime}$ stands for the sum over all $m$ such that

$$
\left(n\left(\log T_{0}\right)^{r}\right)^{\sigma_{2}} \ll\left(d\left(m, T_{0}\right)\right)^{t} .
$$

Proof.

$$
\begin{aligned}
\left(n\left(\log T_{0}\right)^{r}\right)^{2 \sigma_{2}} \sum_{m \in M(\mathbf{T})}^{\prime}\left(d\left(m, T_{0}\right)\right)^{t} & \ll \sum_{m \in M(\mathbf{T})}\left(d\left(m, T_{0}\right)\right)^{3 t} \\
& \ll T_{0}^{n}\left(n\left(\log T_{0}\right)^{r}\right)^{2^{3 t}-1} \ll T_{0}^{n}\left(n\left(\log T_{0}\right)^{r}\right)^{\sigma_{2}} .
\end{aligned}
$$


Proof of Proposition 3.2. We set $G=2^{d-1}$ and get by an application of Lemma 3.7

$$
|S(f, \mathbf{T})|^{G} \ll T^{(G-d) n} \sum_{h_{1}, \ldots, h_{d-1} \in M(2 T)}\left|\sum_{h \in M(\mathbf{T})} E\left(\alpha_{d} m h\right)\right|,
$$

where

$$
m=d ! h_{1} \cdots h_{d-1}
$$

Now we denote by $A(m)$ the number of solutions of $(3.5)$. Noting that $d_{k}(m, T) \leq d(m, T)^{k}$ we get that

$$
A(m) \ll \begin{cases}T^{(d-2) n} & \text { if } m=0, \\ d(m, T)^{d-1} & \text { if } m \neq 0 .\end{cases}
$$

Putting everything together yields

$$
|S(f, \mathbf{T})|^{G} \ll T^{(G-2) n}+T^{(G-d) n} \sum_{m \in M\left(d ! 2^{q} T^{d-1}\right)} d(m, T)^{d-1}\left|\sum_{h \in M(\mathbf{T})} E\left(\alpha_{d} m h\right)\right| .
$$

Now we distinguish two cases for $m$ according to the hypotheses of Lemma 3.11, i.e., whether $\left(n(\log T)^{r}\right)^{\sigma_{2}} \ll d(m, T)$ or not. Thus by an application of Lemma 3.5 and Lemma 3.6

$$
\begin{aligned}
&|S(f, \mathbf{T})|^{G} \ll \\
& T^{(G-2) n}+T^{(G-d) n}\left(T^{n}\left(n(\log T)^{r}\right)^{\sigma}+\sum_{m}^{\prime}\left(n(\log T)^{r}\right)^{\sigma}\left|\sum_{h} E\left(\alpha_{d} m h\right)\right|\right) \\
& \ll T^{(G-2) n}+T^{(G-d+1) n}(\log T)^{r \sigma} \\
&+T^{(G-d) n}(\log T)^{r \sigma} \sum_{m}^{\prime} T^{n-1} \min _{i}\left(T,\left|1-E\left(\alpha_{d} m h\right)\right|^{-1}\right) \\
& \ll T^{(G-2) n}+T^{(G-d+1) n}(\log T)^{r \sigma}+T^{G n}(\log T)^{r \sigma}\left(\frac{1}{\mathrm{~N}\left(\alpha_{d}\right)}+\frac{1}{T}+(\log T)^{-\sigma_{1}}\right) \\
& \ll T^{(G-2) n}+T^{(G-d+1) n}(\log T)^{r \sigma}+T^{G n}(\log T)^{r \sigma-\sigma_{1}} .
\end{aligned}
$$

\section{Fundamental domain}

In this section we want to construct the Urysohn function for indicating the elements starting with a certain digit. The following definitions are standard in that area and we mainly follow Gittenberger and Thuswaldner [11]. We need the fundamental domain, which is defined as the set of all numbers whose integer part is zero, i.e.,

$$
\mathcal{F}^{\prime}:=\left\{z \in \mathbb{C} \mid z=\sum_{k \geq 1} a_{k} b^{-k}, a_{k} \in \mathcal{N}\right\}
$$

It is more convenient to consider the embedding of the fundamental domain in $\mathbb{R}^{n}$. This idea is based on a result by Kovács ( $c f$. Lemma 1 of [18]), that if $(b, \mathcal{N})$ is a CNS then $\left\{1, b, b^{2}, \ldots, b^{n-1}\right\}$ is an integral basis for $K$. Thus we define the embedding $\phi$ by

$$
\phi:\left\{\begin{array}{ccc}
\bar{K} & \rightarrow & \mathbb{R}^{n}, \\
\alpha_{0}+\alpha_{1} b+\cdots+\alpha_{n-1} b^{n-1} & \mapsto\left(\alpha_{0}, \ldots, \alpha_{n-1}\right) .
\end{array},\right.
$$

where $\bar{K}$ is the completition of $K$. 
Now let $m_{b}(x)=a_{0}+a_{1} x+\cdots+a_{n-1} x^{n-1}$ be the minimal polynomial of $b$, then we define the corresponding matrix $B$ by

$$
B=\left(\begin{array}{cccccc}
0 & 0 & \cdots & \ldots & \cdots & -a_{0} \\
1 & 0 & \ldots & \ldots & 0 & \vdots \\
0 & 1 & \ddots & & \vdots & \vdots \\
\vdots & \ddots & \ddots & \ddots & \vdots & \vdots \\
\vdots & & \ddots & 1 & 0 & \vdots \\
0 & 0 & \cdots & 0 & 1 & -a_{n-1}
\end{array}\right)
$$

One easily checks that

$$
\phi(b \cdot z)=B \cdot \phi(z) .
$$

By this we define the embedding of the fundamental domain by

$$
\mathcal{F}=\phi\left(\mathcal{F}^{\prime}\right)=\left\{z \in \mathbb{R}^{n} \mid z=\sum_{k \geq 1} B^{-k} a_{k}, a_{k} \in \phi(\mathcal{N})\right\} .
$$

The rest of this section follows the ideas of Gittenberger and Thuswaldner [11] and therefore we only show the results and left the proofs to the reader. For the construction of the Urysohn function the border of the fundamental domain is of special interest. We can approximate $\mathcal{F}$ with help of the sets

$$
\begin{aligned}
& Q_{0}:=\left\{z \in \mathbb{R}^{2} \mid\|z\|_{\infty} \leq \frac{1}{2}\right\}, \\
& Q_{k}:=\bigcup_{a \in \mathcal{N}} B^{-1}\left(Q_{k-1}+\phi(a)\right) .
\end{aligned}
$$

This approximation satisfies $d\left(\partial Q_{k}, \partial \mathcal{F}\right) \ll|b|^{-k}$, where $d(\cdot, \cdot)$ denotes the Hausdorff metric. By consulting $[25,29]$, we get that the $Q_{k}$ consists of $|\mathcal{N}|^{k}$ parallelograms. Furthermore there exists a $\mu$ with $1<\mu<|\mathrm{N}(b)|$ such that $\mathcal{O}\left(\mu^{k}\right)$ of theses parallelograms intersect the boundary of $Q_{k}$.

Now we define the fundamental domain consisting of all numbers whose first digit equals $a \in \mathcal{N}$, i.e.,

$$
\mathcal{F}_{a}=B^{-1}(\mathcal{F}+\phi(a)) .
$$

Imitating the proof of Lemma 3.1 of [11] we get the following.

Lemma 4.1. For all $a \in \mathcal{N}$ and all $k \in \mathbb{N}$ there exists an axe-parallel tube $P_{k, a}$ with the following properties:

- $\partial \mathcal{F}_{a} \subset P_{k, a}$ for all $k \in \mathbb{N}$,

- the Lebesgue measure of $P_{k, a}$ is a $\mathcal{O}\left(\frac{\mu^{k}}{|\mathrm{~N}(b)|^{k}}\right)$,

- $P_{k, a}$ consists of $\mathcal{O}\left(\mu^{k}\right)$ axe-parallel rectangles, each of which has Lebesgue measure $\mathcal{O}\left(|\mathrm{N}(b)|^{k}\right)$, where $\lambda$ denotes the Lebesgue measure.

As in the proof of Lemma 3.1 of [11] we can construct for each pair $(k, a)$ an axe-parallel polygon $\Pi_{k, a}$ and the corresponding tube

$$
P_{k, a}:=\left\{\left.z \in \mathbb{R}^{n}\left|\left\|z-\Pi_{k, a}\right\|_{\infty} \leq 2 c_{p}\right| b\right|^{-k}\right\} .
$$

Furthermore we denote by $I_{k, a}$ the set of all points inside $\Pi_{k, a}$. Now we define our Urysohn function $u_{a}$ by

$$
u_{a}\left(x_{1}, \ldots, x_{n}\right)=\frac{1}{\Delta^{n}} \int_{-\frac{\Delta}{2}}^{\frac{\Delta}{2}} \cdots \int_{-\frac{\Delta}{2}}^{\frac{\Delta}{2}} \psi_{a}\left(x_{1}+y_{1}, \ldots, x_{n}+y_{n}\right) \mathrm{d} y_{1} \cdots \mathrm{d} y_{n},
$$

where

$$
\Delta:=2 c_{u}|b|^{-k}
$$


and

$$
\psi_{a}\left(x_{1}, \ldots, x_{n}\right)= \begin{cases}1 & \text { if }\left(x_{1}, \ldots, x_{n}\right) \in I_{k, a} \\ \frac{1}{2} & \text { if }\left(x_{1}, \ldots, x_{n}\right) \in \Pi_{k, a} \\ 0 & \text { otherwise }\end{cases}
$$

Thus $u_{a}$ is the desired Urysohn function which equals 1 for $z \in I_{k, a} \backslash P_{k, a}, 0$ for $z \in \mathbb{R}^{n} \backslash\left(I_{k, a} \cup P_{k, a}\right)$, and linear interpolation in between.

We now do a Fourier transform of $u_{a}$ and estimate the coefficients in the same way as in Lemma 3.2 of [11].

Lemma 4.2. Let $u_{a}\left(x_{1}, \ldots, x_{n}\right)=\sum_{\left(m_{1}, \ldots, m_{n}\right) \in \mathbb{Z}^{n}} c_{m_{1}, \ldots, m_{n}} e\left(m_{1} x_{1}+\cdots+m_{n} x_{n}\right)$ be the Fourier expansion of $u_{a}$. Then the Fourier coefficients $c_{m_{1}, \ldots, m_{n}}$ can be estimated by

$$
c_{0, \ldots, 0}=\frac{1}{|\mathrm{~N}(b)|}, \quad c_{m_{1}, \ldots, m_{n}} \ll \mu^{k} \prod_{i=1}^{n} \frac{1}{r\left(m_{i}\right)}
$$

with

$$
r\left(m_{i}\right)= \begin{cases}\Delta m_{i} & m_{i} \neq 0 \\ 1 & m_{i}=0\end{cases}
$$

\section{Estimation of the Border}

Before we proof the central proposition in the next chapter, we have to consider the error term, which mainly comes from the number of points within the tube $P_{k, a}$ defined in the previous chapter. Therefore we fix a positive integer $k$, which we will choose later, and define

$$
F_{j}:=\#\left\{z \in M(\mathbf{T}) \mid \phi\left(\frac{p(z)}{b^{j+1}}\right) \in \bigcup_{a \in \mathcal{N}} P_{k, a} \quad \bmod B^{-1} \mathbb{Z}^{n}\right\} .
$$

The main target of this section is the estimation of $F_{j}$.

Proposition 5.1. Let $\mu<|\mathrm{N}(b)|$ be as in section 4 and $C_{l}$ and $C_{u}$ be sufficiently large positive reals. Suppose that $j$ is a positive integer such that

$$
C_{l} \log \log T \leq j \leq d \log _{\mathrm{N}(b)} T-C_{u} \log \log T .
$$

Then for any positive $\sigma_{3}$

$$
F_{j} \ll \mu^{k} T^{n}\left(|\mathrm{~N}(b)|^{-k}+(\log T)^{-\sigma_{3}}\right) .
$$

In order to estimate $F_{j}$ we apply the Erdős-Turan-Koksma Inequality.

Lemma 5.2 ([6, Theorem 1.21]). Let $x_{1}, \ldots, x_{L}$ be points in the $n$-dimensional real vector space $\mathbb{R}^{n}$ and $H$ an arbitrary positive integer. then the discrepancy $D_{L}\left(x_{1}, \ldots, x_{L}\right)$ fulfills the inequality

$$
D_{L}\left(x_{1}, \ldots, x_{L}\right) \ll \frac{2}{H+1}+\sum_{0<\|\mathbf{h}\|_{\infty} \leq H} \frac{1}{r(\mathbf{h})}\left|\frac{1}{L} \sum_{\ell=1}^{L} e\left(\mathbf{h} \cdot x_{\ell}\right)\right|,
$$

where $\mathbf{h} \in \mathbb{Z}^{n}$ and $r(\mathbf{h})=\prod_{i=1}^{n} \max \left(1,\left|h_{i}\right|\right)$.

Moreover we need that all conjugates of $b$ have absolute value greater than one, which is established by the following lemma.

Lemma 5.3 ([19, Theorem 3]). If $(b, \mathcal{N})$ is a canonical number system, then

$$
\left|b^{(i)}\right|>1 \quad \text { for } 1 \leq i \leq n \text {. }
$$

Now we are ready to prove the proposition. 
Proof of Proposition 5.1. In order to apply the Erdős-Turán-Koksma Inequality we have to consider rectangles. Recall that the tube $P_{k, a}$ consists of rectangles as mentioned in Lemma 4.1. We split the tube $P_{k, a}$ into this family of rectangles and let $R_{a}$ be one of them. Then our goal is to estimate

$$
F_{j}\left(R_{a}\right):=\left\{z \in M(\mathbf{T}) \mid \phi\left(\frac{p(z)}{b^{j+1}}\right) \in R_{a} \bmod B^{-1} \mathbb{Z}^{n}\right\} .
$$

We set $x_{z}:=\phi\left(p(z) / b^{j+1}\right)$ and get by the definition of the discrepancy $(c f$. [6, Definition 1.5$\left.]\right)$ that

$$
F_{j}\left(R_{a}\right) \ll T^{n}\left(\lambda\left(R_{a}\right)+D_{L}\left(\left\{x_{z}\right\}\right)\right),
$$

where $L$ is the number of elements in $M(\mathbf{T})$ and $T$ is the geometric mean of the $T_{i}$. Thus we get by Lemma 3.3 that

$$
L=\frac{2^{r} \pi^{r_{2}}}{\sqrt{D}} T^{n}+\mathcal{O}\left(T^{n-1}\right)
$$

In order to estimate the discrepancy we use Lemma 5.2 to get

$$
D_{L}\left(\left\{x_{z}\right\}\right) \ll \frac{2}{H+1}+\sum_{0<\|\mathbf{h}\|_{\infty} \leq H} \frac{1}{r(\mathbf{h})}\left|\frac{1}{L} \sum_{z \in M(\mathbf{T})} e\left(\mathbf{h} \cdot x_{z}\right)\right| .
$$

Our aim is the application of Proposition 3.2. Since $E$ is defined in (2.1) as $E=e \circ$ Tr we have to rewrite the exponential sum with scalar multiplication into one involving the trace. It is easy to see that the following function suffices our purpose.

$$
\tau(z):=\left(\operatorname{Tr}(z), \operatorname{Tr}(b z), \ldots, \operatorname{Tr}\left(b^{n-1} z\right)\right)=\Xi \phi(z),
$$

where $\Xi=V V^{T}$ and $V$ is the Vandermonde matrix

$$
V=\left(\begin{array}{cccc}
1 & 1 & \cdots & 1 \\
b & b^{(2)} & \cdots & b^{(n)} \\
\vdots & \vdots & & \vdots \\
b^{n-1} & \left(b^{(2)}\right)^{n-1} & \cdots & \left(b^{(n)}\right)^{n-1}
\end{array}\right)
$$

Thus we get

$$
\mathbf{h} \cdot x_{z}=\mathbf{h} \cdot \phi\left(\frac{p(z)}{b^{j-1}}\right)=\mathbf{h} \Xi^{-1} \tau\left(\frac{p(z)}{b^{j-1}}\right)=\operatorname{Tr}\left(\sum_{i=1}^{n} \tilde{h}_{i} b^{i-1} \frac{p(z)}{b^{j-1}}\right)
$$

where we have set $\left(\tilde{h_{1}}, \tilde{h_{2}}, \ldots, \tilde{h_{n}}\right):=\mathbf{h} \Xi^{-1}$.

Plugging (5.4), (5.5) and (5.7) into (5.3) and applying Lemma 4.1 yields

$$
F_{j}\left(R_{a}\right) \ll \lambda\left(R_{a}\right) T^{n}+\frac{T^{n}}{H+1}+\sum_{0<\|\mathbf{h}\|_{\infty} \leq H} \frac{1}{r(\mathbf{h})} \sum_{z \in M(\mathbf{T})} E\left(\sum_{i=1}^{n} \tilde{h}_{i} b^{i-1} \frac{p(z)}{b^{j-1}}\right)
$$

where

$$
H=(\log T)^{\sigma_{4}} .
$$

Now we want to apply Proposition 3.2 for the last sum. Since $\sum_{i=1}^{n} \tilde{h_{i}} b^{i-1} p(z)$ is a polynomial of degree $d$ we write $\xi$ for its leading coefficient for short. Then we apply Lemma 3.1 to get $a \in \delta^{-1}$ and $q \in \mathcal{O}_{K}$ such that

$$
\left|\frac{\xi^{(i)}}{\left(b^{(i)}\right)^{j+1}} q^{(i)}-a^{(i)}\right| \leq T_{i}^{-d}(\log T)^{\sigma_{1}} \quad \text { and } \quad 0<q^{(i)} \leq T_{i}^{d}(\log T)^{-\sigma_{1}} \quad \text { for } \quad 1 \leq i \leq r .
$$

Now we distinguish three cases according to the size of $\mid q$.

- Case 1, $|q| \geq(\log T)^{\sigma_{1}}$ : By Proposition 3.2 we get

$$
\sum_{z \in M(\mathbf{T})} E\left(\sum_{i=1}^{n} \tilde{h}_{i} b^{i-1} \frac{p(z)}{b^{j+1}}\right) \ll T^{n}(\log T)^{-\sigma_{0}} .
$$


- Case 2, $2 \leq|q|<(\log T)^{\sigma_{1}}$ : Let $1 \leq i \leq n$ be such that $\left|q^{(i)}\right| \geq 2$. Then by noting $\left|q^{(i)}\right| \leq \sqrt{q} \mid$ we get

$$
\left|\frac{\xi^{(i)}}{\left(b^{(i)}\right)^{j+1}} q^{(i)}-a^{(i)}\right|<\frac{(\log T)^{\sigma_{1}}}{T_{i}^{d}} \leq \frac{1}{\left|q^{(i)}\right|}
$$

and thus (using our first successive minimum $\lambda_{1}$ )

$$
\left|\frac{\xi^{(i)}}{\left(b^{(i)}\right)^{j+1}}\right| \geq\left|\frac{a^{(i)}}{q^{(i)}}\right|-\frac{1}{\left|q^{(i)}\right|^{2}} \geq \lambda_{1}\left(\frac{1}{\left|q^{(i)}\right|}-\frac{1}{\left|q^{(i)}\right|^{2}}\right) \geq \lambda_{1} \frac{1}{2\left|q^{(i)}\right|} \gg(\log T)^{-\sigma_{1}} .
$$

Therefore by noting Lemma 5.3 and our definition of $H$ in (5.9) we have

$$
\left|b^{(i)}\right|^{j+1} \ll\left|\xi^{(i)}\right|(\log T)^{\sigma_{1}} \ll n H(\log T)^{\sigma_{1}} \ll(\log T)^{\sigma_{1}+\sigma_{4}},
$$

which yields

$$
j \ll \frac{\left(\sigma_{1}+\sigma_{2}\right)}{\log |b|} \log \log T
$$

contradicting the lower bound for $j$ in (5.2) for sufficiently large $C_{l}$.

- Case 3, $0<|q|<2$ : We have to consider two further cases according to whether $a \neq 0$ or $a=0$.

- Case 3.1, $\frac{\xi}{b^{j+1}} q \mid \geq \frac{\lambda_{1}}{2}$ : Let $1 \leq i \leq n$ be such that $\left|\frac{\xi^{(i)}}{\left(b^{(i)}\right)^{j+1}} q^{(i)}\right| \geq \frac{\lambda_{1}}{2}$. Then we get with $H$ as in (5.9)

$$
\left|b^{(i)}\right|^{j+1} \ll H=(\log T)^{\sigma_{4}}
$$

which again contradicts the lower bound of $j$ in (5.2).

- Case 3.2, $\frac{\xi}{b^{j+1}} q \mid<\frac{\lambda_{1}}{2}$ : Since $\lambda_{1}$ is the first successive minima for the sphere with respect to the lattice of $\delta^{-1}$ we get that $a=0$. Thus for $1 \leq i \leq n$

$$
\left|\frac{\xi^{(i)}}{\left(b^{(i)}\right)^{j+1}} q^{(i)}\right|<\frac{(\log T)^{\sigma_{1}}}{T_{i}^{d}} .
$$

Talking the norm we get

$$
\mathrm{N}\left(\frac{\xi}{b^{j+1}} q\right)=\frac{\mathrm{N}(\xi)}{\mathrm{N}\left(b^{j+1}\right)} \mathrm{N}(q)<\frac{(\log T)^{n \sigma_{1}}}{T^{n d}} .
$$

This implies

$$
j+1 \geq n d \log _{|\mathrm{N}(b)|} T-\frac{n \sigma}{\log |\mathrm{N}(b)|} \log \log T+C
$$

contradicting the upper bound of $j$ in (5.2) for sufficiently large $C_{u}$.

Thus we get for all $j$ satisfying (5.2), that

$$
\sum_{z \in M(\mathbf{T})} E\left(\sum_{i=1}^{n} \tilde{h}_{i} b^{i-1} \frac{f(z)}{b^{j+1}}\right) \ll T^{n}(\log T)^{-\sigma_{0}} .
$$

Plugging this into (5.8) together with (5.9) yields

$$
\begin{aligned}
F_{j}\left(R_{a}\right) & \ll T^{n} \lambda\left(R_{a}\right)+\frac{T^{n}}{(\log T)^{\sigma_{4}}}+T^{n}(\log T)^{-\sigma_{0}} \sum_{0<\|\mathbf{h}\|_{\infty} \leq H} \frac{1}{r(\mathbf{h})} \\
& \ll T^{n} \lambda\left(R_{a}\right)+\frac{T^{n}}{(\log T)^{\sigma_{4}}}+T^{n}(\log T)^{-\sigma_{0}}(\log \log T)^{n} .
\end{aligned}
$$

Finally setting $\sigma_{4}:=\sigma_{0} / 2$ and summing over all rectangles $R_{a}$ yields

$$
F_{j} \ll \mu^{k} T^{n}\left(|\mathrm{~N}(b)|^{-k}+(\log T)^{-\sigma_{0} / 2}\right) .
$$

By setting $\sigma_{3}=\sigma_{0} / 2$ the proposition is proven. 


\section{THE CENTRAL PROPOSITION}

Before we can state the central proposition we need a lemma estimating the length of the expansion of every integer. This will provide us with an upper bound for the scope of interest (namely for the $T_{i}$ in $M(\mathbf{T})$ ) and further establish us with the tools needed in order to prove the Theorem 2.1 .

Lemma 6.1 ([20, Theorem]). Let $\ell(\gamma)$ be the length of the expansion of $\gamma$ to the base $b$. Then

$$
\left|\ell(\gamma)-\max _{1 \leq i \leq n} \frac{\log \left|\gamma^{(i)}\right|}{\log \left|b^{(i)}\right|}\right| \leq C .
$$

We fix a $T$ and set $T_{i}$ for $1 \leq i \leq n$ such that

$$
\log T_{i}=\log T \frac{\log \left|b^{(i)}\right|^{n}}{\log |\mathrm{N}(b)|}
$$

Thus we get in view of Lemma 6.1 that the expansions of the elements of $M(\mathbf{T})$ have the same maximum length. Now we are in the position to state the central proposition in the proof of Theorem 2.1 .

Proposition 6.2. Let $T \geq 0$ and $T_{i}$ for $1 \leq i \leq n$ be defined as in (6.1). Let $L$ be the maximum length of the b-adic expansion of $z \in M(\mathbf{T})$ and let $C_{l}$ and $C_{u}$ be sufficiently large. Then for

$$
C_{l} \log L \leq l_{1}<l_{2}<\cdots<l_{h} \leq d L-C_{u} \log L
$$

we have,

$$
\Theta:=\#\left\{z \in M(\mathbf{T}) \mid a_{l_{j}}(f(z))=b_{j}, j=1, \ldots, h\right\}=\frac{2^{r} \pi^{r_{2}}}{\sqrt{D}|\mathrm{~N}(b)|^{h}} T^{n}+\mathcal{O}\left(T^{n}(\log T)^{-\sigma_{0}}\right)
$$

uniformly for $T \rightarrow \infty$, where $b_{j} \in \mathcal{N}$ are given and $\sigma_{0}$ is an arbitrary positive constant.

Proof. We recall our Urysohn function $u_{a}$ (defined in (4.1)) and set for $\nu \in \mathbb{R}^{n}$

$$
t(\nu)=u_{b_{1}}\left(B^{-l_{1}-1} \nu\right) \cdots u_{b_{h}}\left(B^{-l_{h}-1} \nu\right) .
$$

Now we want to apply the Fourier transformation, which we developed in Lemma 4.2. Therefore we set

$$
\mathcal{M}:=\left\{M=\left(\mu_{1}, \ldots, \mu_{h}\right) \mid \mu_{j}=\left(m_{j 1}, \ldots, m_{j n}\right) \in \mathbb{Z}^{n}, \text { for } j=1, \ldots, h\right\} .
$$

An application of Lemma 4.2 yields

$$
t(\nu)=\sum_{M \in \mathcal{M}} T_{M} e\left(\sum_{j=1}^{h} \mu_{j} B^{-l_{j}-1} \nu\right),
$$

where $T_{M}=\prod_{j=1}^{h} c_{m_{j 1}, \ldots, m_{j n}}$. Combining these results we get

$$
\left|\Theta-\sum_{z \in M(\mathbf{T})} t(\phi(p(z)))\right| \leq F_{l_{1}}+\cdots+F_{l_{h}} .
$$

Using the function $\tau$ defined in (5.6) we get

$$
\sum_{z \in M(\mathbf{T})} t(\phi(p(z)))=\sum_{M \in \mathcal{M}} T_{M} \sum_{z \in M(\mathbf{T})} e\left(\sum_{j=1}^{h} \mu_{j} B^{-l_{j}-1} \Xi^{-1} \tau(p(z))\right) .
$$

Setting

yields

$$
\tilde{\mu_{j}}=\left(\tilde{m_{j 1}}, \ldots, \tilde{m_{j n}}\right):=\mu_{j} B^{-l_{j}-1} \Xi^{-1} \quad(j=1, \ldots, h)
$$

$$
\sum_{z \in M(\mathrm{~T})} t(\phi(p(z)))=\sum_{M \in \mathcal{M}} T_{M} \sum_{z \in M(\mathbf{T})} E\left(\sum_{j=1}^{h} \sum_{i=1}^{n} \frac{\left.\tilde{m}_{j i} p(z)\right)}{b^{l_{j}+1}}\right) .
$$


We want to apply the same ideas as in the proof of Proposition 5.1. For $1 \leq j \leq h$ we set $\xi_{j}$ to be the leading coefficient of $\sum_{i=1}^{n} \tilde{m}_{j i} p(z)$. Then we apply Lemma 3.1 with $N_{i}=T_{i}^{d}(\log T)^{-\sigma_{1}}$ for $1 \leq i \leq r$ in order to get that there exist $a \in \delta^{-1}$ and $q \in \mathcal{O}_{K}$ such that

$$
\left|\sum_{j=1}^{h} \frac{\xi_{j}^{(i)}}{\left(b^{(i)}\right)^{l_{j}+1}} q^{(i)}-a^{(i)}\right|<\frac{(\log T)^{\sigma_{1}}}{T_{i}^{d}} \quad \text { and } \quad 0<\left|q^{(i)}\right|<\frac{T_{i}^{d}}{(\log T)^{\sigma_{1}}} \quad \text { for } \quad 1 \leq i \leq n .
$$

Now we again distinguish several cases.

- Case 1, $|q| \geq(\log T)^{\sigma_{1}}$ : We apply Proposition 3.2 and get

$$
\sum_{z \in M(\mathbf{T})} E\left(\sum_{j=1}^{h} \sum_{i=1}^{n} \frac{\left.\tilde{m}_{j i} p(z)\right)}{b^{l_{j}+1}}\right) \ll T^{n}(\log T)^{-\sigma_{0}}
$$

- Case 2, $2 \leq|q|<(\log T)^{\sigma_{1}}$ : In the same manner as in (5.10) we get

$$
\begin{aligned}
(\log T)^{\sigma_{1}} \leq & \left|\sum_{j=1}^{h} \frac{\xi_{j}^{(i)}}{\left(b^{(i)}\right)^{l_{j}+1}}\right| \leq \frac{\sum_{j=1}^{h}\left|\xi_{j}^{(i)}\right|}{\left|b^{(i)}\right|^{l_{1}+1}}, \\
& l_{1}+1 \ll \log \log T .
\end{aligned}
$$

Together with the definition of $L$ this contradicts the lower bound of $l_{1}$ for sufficiently large $C_{l}$ in $(6.2)$.

- Case 3, $0<|q|<2$ : We have to consider two sub cases

- Case 3.1, $\mid \sum_{j=1}^{h} \frac{\xi_{j}}{b^{h_{j}+1}} q \geq \frac{\lambda_{1}}{2}$ : Let $1 \leq i \leq n$ be such that

$$
\frac{\lambda_{1}}{2} \leq\left|\sum_{j=1}^{h} \frac{\xi_{j}^{(i)}}{\left(b^{(i)}\right)^{l_{j}+1}} q^{(i)}\right| \leq \frac{\sum_{j=1}^{h} \mid \xi_{j}^{(i)}}{\left|b^{(i)}\right|^{l_{1}+1}}\left|q^{(i)}\right|
$$

then

$$
l_{1}+1 \ll \log \log T
$$

again contradicts the lower bound of $l_{1}$ for sufficiently large $C_{l}$ in (6.2).

- Case 3.2, $\left|\sum_{j=1}^{h} \frac{\xi_{j}}{b^{l_{j}+1}} q\right|<\frac{\lambda_{1}}{2}$ : By Minkowski's theorem we get that $a=0$. Thus for $1 \leq i \leq n$

$$
\left|\sum_{j=1}^{h} \frac{\xi_{j}^{(i)}}{\left(b^{(i)}\right)^{l_{j}+1}} q^{(i)}\right|=\left|\frac{1}{\left(b^{(i)}\right)^{l_{h}+1}} \sum_{j=1}^{h} \xi_{j}^{(i)}\left(b^{(i)}\right)^{l_{h}-l_{j}} q^{(i)}\right| \leq \frac{(\log T)^{\sigma_{1}}}{T_{i}^{d}}
$$

which implies (taking the norm of the left side)

$$
l_{h}+1 \geq n d \log _{|\mathrm{N}(b)|} T-c\left(\log \log _{|\mathrm{N}(b)|} T\right)
$$

contradicting the upper bound for sufficiently large $C_{u}$.

After these considerations it is clear, that Case $\mathbf{1}$ is the only possible case which suffices the hypotheses in (6.2). Plugging this into (6.3) and applying Lemma 4.2 and Lemma 3.3 for the coefficient $c_{0, \ldots, 0}$ yields

$$
\Theta=\frac{2^{r} \pi^{r_{2}}}{\sqrt{D}|\mathrm{~N}(b)|^{h}} T^{n}+\mathcal{O}\left(T^{n}(\log T)^{-\sigma_{0}} \sum_{0 \neq M \in \mathcal{M}} T_{M}\right)+\mathcal{O}\left(\sum_{j=1}^{h} F_{j}\right) .
$$

An application of Proposition 5.1 with $k \ll \log \log T$ and the observation that

$$
\sum_{M \in \mathcal{M}}\left|T_{M}\right| \ll \Delta^{-2 h} \ll|b|^{2 h k} \ll(\log T)^{\sigma_{0} / 2},
$$

where we used the definition of $\Delta$ in (4.2), proves the proposition. 


\section{Proof of Theorem 2.1}

At this point we will need the Fréchet-Shohat Theorem which we state for completeness.

Lemma $7.1\left(\left[9\right.\right.$, Lemma 1.43]). Let $\left(F_{n}(z)\right)_{n \geq 1}$ be a sequence of distribution functions. For each non-negative integer $k$ let

$$
\alpha_{k}=\lim _{n \rightarrow \infty} \int_{-\infty}^{\infty} z^{k} \mathrm{~d} F_{n}(z)
$$

exist.

Then there is a subsequence of $F_{n_{j}}(z)\left(n_{1}<n_{2}<\cdots\right)$, which converges weakly to a limiting distribution $F(z)$ for which

$$
\alpha_{k}=\int_{-\infty}^{\infty} z^{k} \mathrm{~d} F(z) \quad(k=0,1, \ldots) .
$$

Moreover, if the set of moments $\alpha_{k}$ determine $F(z)$ uniquely, then as $n \rightarrow \infty$ the distribution $F_{n}(z)$ converge weakly to $F(z)$.

For the proof of Theorem 2.1 we mainly follow the proof of Theorem 1.2 of Gittenberger and Thuswaldner [11]. In the same manner as in their proof we set $C:=\max \left(C_{l}, C_{u}\right), A:=[C \log L]$ and $B:=L-A$, where $L, C_{l}$ and $C_{u}$ are defined in the statement of Proposition 6.2. Furthermore we define the truncated function $f^{\prime}$ to be

$$
f^{\prime}(p(z))=\sum_{j=A}^{B} f\left(a_{j}(p(z)) b^{j}\right) .
$$

By the definition of $A$ and $f\left(a b^{j}\right) \ll 1$ with $a \in \mathcal{N}$ we get that $f^{\prime}(p(z))=f(p(z))+\mathcal{O}(\log L)$. In the same manner we define the truncated mean and standard deviation

$$
M_{b}^{\prime}(T):=\sum_{j=A}^{B} m_{j} \quad \text { and } \quad D_{b}^{\prime 2}(T):=\sum_{j=A}^{B} \sigma_{j}^{2} .
$$

Since $M_{b}(T)-M_{b}^{\prime}(T)=\mathcal{O}(\log L)$ and $D_{b}^{2}(T)-D_{b}^{\prime 2}(T)=\mathcal{O}(\log L)$ we get that it suffices to show that

$$
\frac{1}{\# M(T)} \#\left\{z \in M(T) \mid \frac{f^{\prime}(p(z))-M_{b}^{\prime}\left(T^{d}\right)}{D_{b}^{\prime}\left(T^{d}\right)}<y\right\} \longrightarrow \Phi(y) .
$$

By Lemma 7.1 this holds true if and only if the moments

$$
\xi_{k}(T):=\frac{1}{\# M(T)} \sum_{z \in M(T)}\left(\frac{f^{\prime}(p(z))-M_{b}^{\prime}\left(T^{d}\right)}{D_{b}^{\prime}\left(T^{d}\right)}\right)^{k}
$$

converge to the moments of the normal law for $T \rightarrow \infty$. We will show the last statement by comparing the moments $\xi_{k}$ with

$$
\eta_{k}(T):=\frac{1}{\# M\left(T^{d}\right)} \sum_{z \in M\left(T^{d}\right)}\left(\frac{f^{\prime}(z)-M_{b}^{\prime}\left(T^{d}\right)}{D_{b}^{\prime}\left(T^{d}\right)}\right)^{k} .
$$

An application of Proposition 6.2 gives that

$$
\xi_{k}(T)-\eta_{k}(T) \rightarrow 0 \text { for } T \rightarrow \infty .
$$

Furthermore we get by Lemma 6.1 that these sums consist of independently identically distributed random variables (with possible $2 C$ exceptions). By the central limit theorem we get that their distribution converges to the normal law. Thus the $\eta_{k}(T)$ converge to the moments of the normal law. This yields

$$
\lim _{T \rightarrow \infty} \xi_{k}(T)=\lim _{T \rightarrow \infty} \eta_{k}(T)=\int x^{k} \mathrm{~d} \Phi .
$$

We apply the Fréchet-Shohat Theorem again to prove the theorem. 


\section{ACKNOWLEDGEMENT}

Parts of the work were written when the author was at "laboratoire GREYC" of University of Caen.

\section{REFERENCES}

[1] Shigeki Akiyama, Horst Brunotte, and Attila Pethő, Cubic CNS polynomials, notes on a conjecture of W. J. Gilbert, J. Math. Anal. Appl. 281 (2003), no. 1, 402-415.

[2] N. L. Bassily and I. Kátai, Distribution of the values of q-additive functions on polynomial sequences, Acta Math. Hungar. 68 (1995), no. 4, 353-361.

[3] Horst Brunotte, Andrea Huszti, and Attila Pethő, Bases of canonical number systems in quartic algebraic number fields, J. Théor. Nombres Bordeaux 18 (2006), no. 3, 537-557.

[4] H. Delange, Sur la fonction sommatoire de la fonction "somme des chiffres", Enseignement Math. (2) 21 (1975), no. 1, 31-47.

[5] M. Drmota and J. Gajdosik, The distribution of the sum-of-digits function, J. Théor. Nombres Bordeaux 10 (1998), no. 1, 17-32.

[6] M. Drmota and R. F. Tichy, Sequences, discrepancies and applications, Lecture Notes in Mathematics, vol. 1651, Springer-Verlag, Berlin, 1997.

[7] J. M. Dumont, P. J. Grabner, and A. Thomas, Distribution of the digits in the expansions of rational integers in algebraic bases, Acta Sci. Math. (Szeged) 65 (1999), no. 3-4, 469-492.

[8] J. M. Dumont and A. Thomas, Gaussian asymptotic properties of the sum-of-digits function, J. Number Theory 62 (1997), no. 1, 19-38.

[9] P. D. T. A. Elliott, Probabilistic number theory. I, Grundlehren der Mathematischen Wissenschaften [Fundamental Principles of Mathematical Science], vol. 239, Springer-Verlag, New York, 1979, Mean-value theorems.

[10] B. Gittenberger and J. M. Thuswaldner, The moments of the sum-of-digits function in number fields, Canad. Math. Bull. 42 (1999), no. 1, 68-77.

[11] _ Asymptotic normality of b-additive functions on polynomial sequences in the Gaussian number field, J. Number Theory 84 (2000), no. 2, 317-341.

[12] P. J. Grabner, P. Kirschenhofer, and H. Prodinger, The sum-of-digits function for complex bases, J. London Math. Soc. (2) 57 (1998), no. 1, 20-40.

[13] L.-K. Hua, Additive Primzahltheorie, B. G. Teubner Verlagsgesellschaft, Leipzig, 1959.

[14] I. Kátai and I. Környei, On number systems in algebraic number fields, Publ. Math. Debrecen 41 (1992), no. 3-4, 289-294.

[15] I. Kátai and B. Kovács, Kanonische Zahlensysteme in der Theorie der quadratischen algebraischen Zahlen, Acta Sci. Math. (Szeged) 42 (1980), no. 1-2, 99-107.

[16] _ Canonical number systems in imaginary quadratic fields, Acta Math. Acad. Sci. Hungar. 37 (1981), no. 1-3, 159-164.

[17] I. Kátai and P. Liardet, Additive functions with respect to expansions over the set of Gaussian integers, Acta Arith. 99 (2001), no. 2, 173-182.

[18] B. Kovács, Canonical number systems in algebraic number fields, Acta Math. Acad. Sci. Hungar. 37 (1981), no. 4, 405-407.

[19] B. Kovács and A. Pethö, Number systems in integral domains, especially in orders of algebraic number fields, Acta Sci. Math. (Szeged) 55 (1991), no. 3-4, 287-299.

[20] . On a representation of algebraic integers, Studia Sci. Math. Hungar. 27 (1992), no. 1-2, 169-172.

[21] M. G. Madritsch, Generating normal numbers over gaussian integers, Acta Arith., to appear.

[22] __ A note on normal numbers in matrix number systems, Math. Pannon. 18 (2007), no. 2, 219-227.

[23] C. Mauduit and A. Sárközy, On the arithmetic structure of the integers whose sum of digits is fixed, Acta Arith. 81 (1997), no. 2, 145-173.

[24] T. Mitsui, On the Goldbach problem in an algebraic number field. I, II, J. Math. Soc. Japan 12 (1960), 290-324, $325-372$.

[25] W. Müller, J. M. Thuswaldner, and R. F. Tichy, Fractal properties of number systems, Period. Math. Hungar. 42 (2001), no. 1-2, 51-68.

[26] A. Pethö, On a polynomial transformation and its application to the construction of a public key cryptosystem, Computational number theory (Debrecen, 1989), de Gruyter, Berlin, 1991, pp. 31-43.

[27] A. Pethő and R. F. Tichy, S-unit equations, linear recurrences and digit expansions, Publ. Math. Debrecen 42 (1993), no. 1-2, 145-154.

[28] P. Ribenboim, Classical theory of algebraic numbers, Universitext, Springer-Verlag, New York, 2001.

[29] K. Scheicher and J. M. Thuswaldner, Canonical number systems, counting automata and fractals, Math. Proc. Cambridge Philos. Soc. 133 (2002), no. 1, 163-182.

[30] _ On the characterization of canonical number systems, Osaka J. Math. 41 (2004), no. 2, 327-351.

[31] Carl Ludwig Siegel, Generalization of Waring's problem to algebraic number fields, Amer. J. Math. 66 (1944), 122-136. 
[32] J. M. Thuswaldner, The sum of digits function in number fields, Bull. London Math. Soc. 30 (1998), no. 1, $37-45$.

[33] _ The sum of digits function in number fields: distribution in residue classes, J. Number Theory $\mathbf{7 4}$ (1999), no. 1, 111-125.

[34] J. M. Thuswaldner and R. F. Tichy, Waring's problem with digital restrictions, Israel J. Math. 149 (2005), 317-344, Probability in mathematics.

[35] Y. Wang, Diophantine equations and inequalities in algebraic number fields, Springer-Verlag, Berlin, 1991.

(M. G. Madritsch) Institute of Statistics, Graz University of Technology, A-8010 Graz, Austria E-mail address: madritsch@finanz.math.tugraz.at 